\title{
Clinicopathologic and genetic characteristics of young patients with pleural diffuse malignant mesothelioma
}

\author{
Marina Vivero ${ }^{1}$, Raphael Bueno ${ }^{2}$ and Lucian R Chirieac ${ }^{1}$ \\ ${ }^{1}$ Department of Pathology, Brigham and Women's Hospital, Harvard Medical School, Boston, MA, USA and \\ ${ }^{2}$ Department of Surgery, Brigham and Women's Hospital, Harvard Medical School, Boston, MA, USA
}

\begin{abstract}
Pleural diffuse malignant mesothelioma typically presents during the seventh decade of life and has poor prognosis. Recent epidemiologic studies have shown differences between young and older mesothelioma patients, but the biology of pleural mesothelioma in young patients is poorly understood. We studied the clinicopathologic and genetic characteristics in pleural mesothelioma patients aged 35 years and younger. Thirty-six consecutive pleural mesothelioma patients aged 35 years and younger were compared with 48 older patients. We examined demographic and clinical characteristics, histologic type, growth patterns, mitotic index, and nuclear grade on hematoxylin and eosin-stained slides, BAP1 protein expression by immunohistochemistry, and CDKN2A and NF2 deletions by fluorescence in situ hybridization. Clinicopathologic and cytogenetic results were compared between young and older groups, and correlated with overall survival. Young patients were more frequently women, reported less asbestos exposure, and had a greater frequency of prior therapeutic radiation and family history of breast cancer than older patients $(P<0.05$ each). There were no histologic differences between young and older patients (all $P>0.05$ ). CDKN2A deletion was less prevalent in young patients $(P=0.01)$, loss of BAP1 protein expression less frequent in young patients $(P=0.06)$, and $N F 2$ deletion rates similar between groups $(P>0.05$ each). Median overall survival was 40 vs 26 months $(P=0.10)$ in young and older patients, respectively, and 47 vs 31 months $(P=0.04)$ when comparing patients with epithelioid histology only. High mitotic index and non-epithelioid histology were the only characteristics associated with a poor overall survival in young patients. Young patients with pleural mesothelioma have an equal sex distribution and are more likely to have a history of mantle radiation, family history of breast cancer, and lower rates of CDKN2A deletion than older patients. Our results suggest that pleural mesothelioma in young patients has distinctive clinical and genetic characteristics, despite some similarities to pleural mesothelioma in older patients.
\end{abstract}

Modern Pathology (2018) 31, 122-131; doi:10.1038/modpathol.2017.108; published online 8 September 2017

Pleural diffuse malignant mesothelioma is an aggressive malignancy of mesothelial derivation that has been etiologically linked to occupational and environmental asbestos exposure in $50-80 \%$ of cases. ${ }^{1-3}$ The median age at diagnosis is 69 years, and the male to female ratio is approximately 3:1 among all patients with pleural mesothelioma. ${ }^{4-6}$ The divergence in sex distribution among patients with mesothelioma occurs between the ages of 35 and $40 .{ }^{6-8}$ In contrast to older individuals with pleural

Correspondence: Dr M Vivero, MD, Department of Pathology, Brigham and Women's Hospital, Harvard Medical School, Amory 3, 75 Francis Street, Boston, MA 02115, USA.

E-mail: mvivero@partners.org

Received 2 March 2017; revised 5 July 2017; accepted 6 July 2017; published online 8 September 2017 mesothelioma, the sex distribution among young pleural mesothelioma patients is approximately equal, and the rates of reported asbestos exposure among this population have been significantly lower than in older patients. ${ }^{8-11}$ These differences in demographics and exposure history between younger and older patients suggest that the etiologic, clinicopathologic, and genetic characteristics of pleural mesothelioma in this population may be unique and distinct from the characteristics of pleural mesothelioma in older individuals. ${ }^{7,11}$

Pleural mesothelioma is rare in young adults, and patients younger than 35 represented $0.7 \%$ of all cases in the National Cancer Institute's Surveillance, Epidemiology, and End Results Program database between 2008 and 2012.6,7,9 Although epidemiologic studies have addressed some clinical characteristics 
of pleural mesothelioma in younger individuals, direct histopathologic studies of tumors from younger patients are few. Among case reports and small series focusing on pediatric patients, the largest to date comprises only 13 patients with histologically confirmed mesothelioma. ${ }^{7,9,10,12-14}$

Given that the prevalence of mesothelioma rises sharply after the age of $35,{ }^{6,7}$ we studied patients aged 35 and younger to characterize this group of patients and determine if their clinical, histologic, and genetic characteristics differ from older patients. We compared clinical features, history of asbestos exposure, personal and familial history of malignancy, CDKN2A and NF2 deletion, and BAP1 protein expression between young and older patients, and compared the results with overall survival.

\section{Materials and methods}

\section{Patient Selection and Clinical Characteristics}

Cases of pleural diffuse malignant mesothelioma were identified and analyzed retrospectively with approval of the Brigham and Women's Hospital Institutional Review Board. Consecutive patients with pleural mesothelioma aged 35 and younger who were seen at Brigham and Women's Hospital between January 1990 and September 2015 were included in the study group. A separate cohort of pleural mesothelioma patients older than 35 treated between April 2012 and September 2015 were used as a comparison group for clinical, histologic, and genetic correlative analyses. This group was selected on the basis of availability of next-generation sequencing results, which was implemented routinely at Brigham and Women's Hospital in 2013 and performed on selected cases from 2012, in order to validate the use of BAP1 immunohistochemistry for determination of BAP1 gene status in the study group, who did not have available gene sequencing results. Information regarding sex, age, history of mantle radiation, asbestos exposure, personal history of malignancy, and family history of malignancy was obtained from the medical records.

\section{Histology Review}

Available hematoxylin and eosin slides and immunohistochemical stains were reviewed for each case in the study group and comparison group. Histologic type, lymphovascular invasion, lymph node metastases, predominant growth pattern, nuclear grade as previously described, ${ }^{15}$ and mitotic index, defined as number of mitoses per $2 \mathrm{~mm}^{2}$ (using an Olympus BX 41 microscope at $\times 400$ magnification, objective $\times 40$, visible area $2.37 \mathrm{~mm}^{2}$ ) were recorded. For purposes of statistical analysis, mitotic index was categorized in binary fashion as $\leq 4$ or $>4$, as this threshold has been previously associated with poor survival. ${ }^{15}$

\section{Cytogenetic Analysis and Fluorescence In Situ Hybridization}

Cytogenetic analysis and fluorescence in situ hybridization to evaluate for CDKN2A (p16) and NF2 deletions were performed as part of routine clinical practice at BWH, as previously published. ${ }^{16}$ Karyotypic abnormalities and CDKN2A and NF2 FISH results were obtained from patient medical records when available.

\section{BAP1 Immunohistochemistry and Next-Generation Sequencing}

Immunohistochemical evaluation of BAP1 protein expression was performed following pressure cooker antigen retrieval (0.01 M citrate buffer, $\mathrm{pH}$ 6.0) using a mouse monoclonal antibody to BAP1 (1:50 dilution; clone C-4; Santa Cruz Biotechnology, Dallas, TX, USA) and the Dako Envision+ detection system (Agilent Technologies, Santa Clara, CA, USA). Staining was performed on 4- $\mu$ m-thick formalin-fixed paraffin-embedded tissue sections. Appropriate positive and negative controls were used throughout the study, and endogenous levels of BAP1 staining in lymphocytes and stromal cells served as internal positive controls in all cases. Intensity of nuclear staining was characterized as absent (0), weak (1+), moderate $(2+)$, or strong $(3+)$, and the extent of immunoreactivity was quantified as percentage of tumor cells staining, in increments of $10 \%$. Examples of BAP1 staining are illustrated in Figure 1.

Systematic evaluation of BAP1 immunohistochemistry in relation to $B A P 1$ gene mutations, copy number variations, and structural rearrangements has not been published, and we therefore correlated BAP1 IHC in the comparison group with next-generation sequencing results in order to validate its use as an indicator of gene status. The OncoPanel targeted hybridcapture-based next generation sequencing assay was performed at Brigham and Women's Hospital after DNA isolation from formalin-fixed paraffin-embedded tissue sections containing more than 20\% tumor using a solution-phase Agilent SureSelect hybrid capture kit and Illumina HiSeq 2500 sequencer as previously described. ${ }^{17}$ Massively parallel sequencing was used to survey exonic DNA sequences from 275 cancer-related genes including CDKN2A, NF2, and $B A P 1$, and 113 intronic sequences from 30 genes, including $B A P 1$, to detect large deletions and gene rearrangements. Sample reads were analyzed and interpreted using a custom pipeline, including mutation calls generated using MuTect ${ }^{18}$ and GATK, copy number calls using VisCap Cancer, ${ }^{19}$ and structural variants using BreakMer. ${ }^{20}$

\section{Statistics}

Categorical data were analyzed using the Fisher Exact Test or $\chi^{2}$ analysis where appropriate, with a 

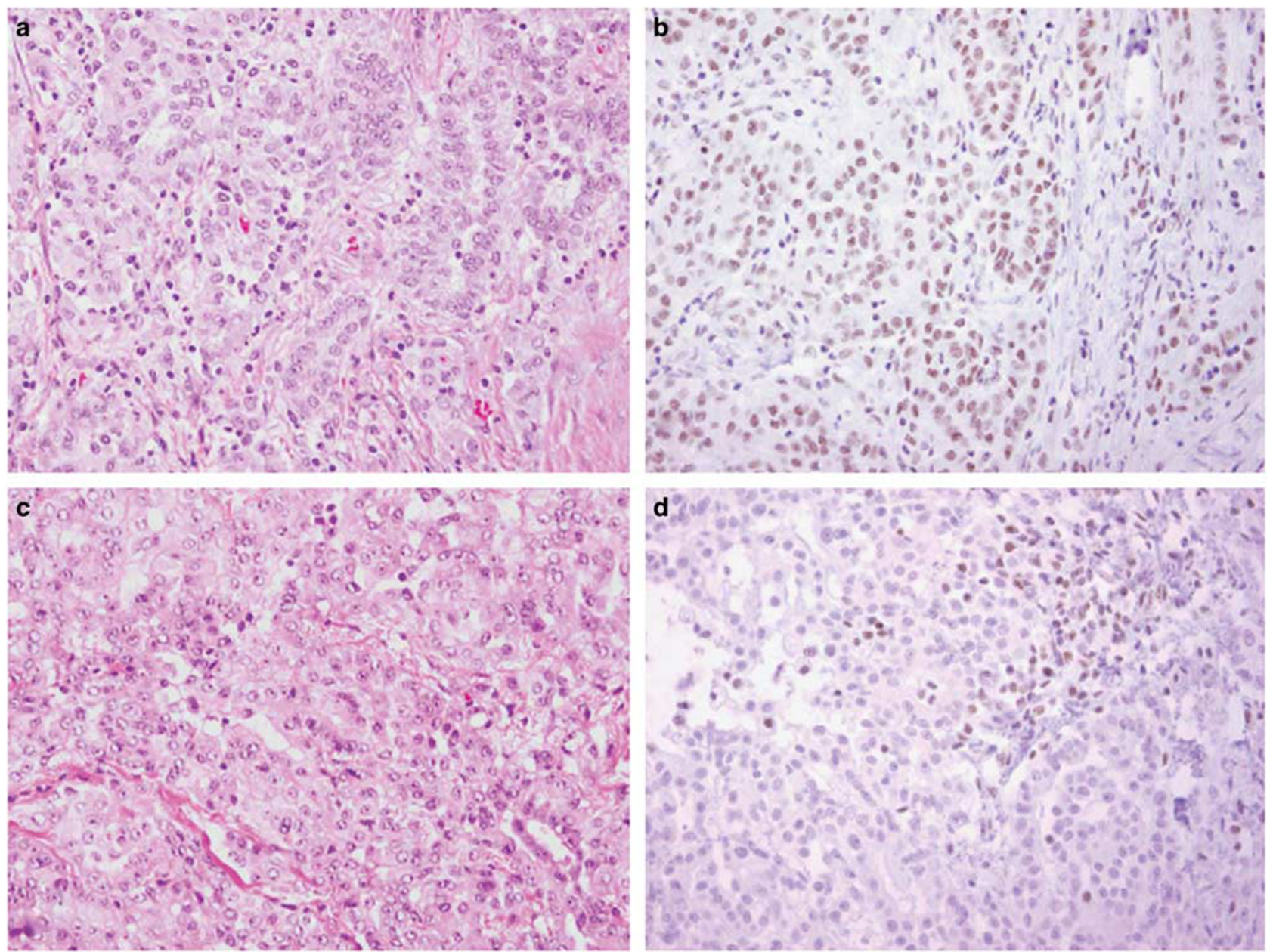

Figure 1 Corresponding H\&E slides and BAP1 immunohistochemistry in young patients with retention (a,b) and complete absence of (c, d) nuclear BAP1 protein expression. Nuclear staining seen in tumor-infiltrating lymphocytes serves as an internal control of staining in panel (d). All images taken at $\times 200$ magnification.

threshold of $P<0.05$ used to determine statistical significance. Overall survival was calculated from the date of pathologic diagnosis to the date of last clinical follow-up or death, at which point the survival data were censored. Median overall survival was determined using the Kaplan-Meier method. Prognostic significance of clinicopathologic characteristics within the study group was determined using Cox regression analysis. Statistical analysis was performed using GraphPad Software (GraphPad In Stat http://www.graphpad.com; version 3.10 for Windows; LaJolla, CA, USA).

\section{Results}

\section{Patient Clinical Characteristics}

We identified 39 consecutive patients with pleural diffuse malignant mesothelioma aged 35 and younger between January 1999 and September 2015, of which 36 had sufficient material for further study (study group). A separate cohort of 48 patients older than 35 with available next-generation sequencing results obtained between April 2012 and September 2015 were used for clinical, histologic and genetic comparison (comparison group). The clinical characteristics of study patients are summarized in Table 1.

The young pleural mesothelioma group consisted of $17(47 \%)$ women and $19(53 \%)$ men, in contrast to the older pleural mesothelioma group, which had a much smaller proportion of women (13\%; $P=0.0005$ ). Mean ages were 30 (SD 4.5; range 18-35) in the young group and 69 (SD 8.3; range 38-84) in the older group. Eight (22\%) young patients had a history of therapeutic mantle radiation, all for Hodgkin lymphoma, while no older patients had received radiation $(P=0.0007)$. Reported asbestos exposure was less common in young patients compared with older patients $(31 \%$ vs $75 \%$; $P=0.0001$ ). While younger patients were as likely to have a personal history of malignancy as older patients ( $36 \%$ vs $27 \%$; $P=0.48$ ), they were less likely to have a family history of malignancy ( $42 \%$ vs $73 \%$; $P=0.003$ ). Analysis of family history of malignancy 
Table 1 Clinical characteristics of study patients

\begin{tabular}{|c|c|c|c|}
\hline Characteristic & $\begin{array}{c}\text { Patients } \leq 35 \\
\mathrm{~N}=36(\%)\end{array}$ & $\begin{array}{c}\text { Patients }>35 \\
\mathrm{~N}=48(\%)\end{array}$ & $\mathrm{P}$-value \\
\hline \multicolumn{4}{|l|}{ Sex } \\
\hline $\mathrm{M}$ & $19(53)$ & $42(87)$ & \multirow[t]{2}{*}{0.0005} \\
\hline $\mathrm{F}$ & $17(47)$ & $6(13)$ & \\
\hline \multicolumn{4}{|l|}{ Age } \\
\hline Mean & 30 & 69 & \multirow{2}{*}{$<0.0001$} \\
\hline Range & $18-35$ & $38-84$ & \\
\hline \multicolumn{4}{|c|}{ History of mantle radiation } \\
\hline Present & $8(22)$ & $0(0)$ & \multirow[t]{2}{*}{0.0007} \\
\hline Absent & $28(78)$ & $48(100)$ & \\
\hline \multicolumn{4}{|c|}{ Asbestos exposure } \\
\hline Present & $11(31)$ & $36(75)$ & \multirow[t]{2}{*}{0.0001} \\
\hline Absent & $25(69)$ & $12(25)$ & \\
\hline \multicolumn{4}{|c|}{ Personal history of malignancy } \\
\hline Present & $13(36)$ & $13(27)$ & \multirow[t]{2}{*}{0.48} \\
\hline Absent & $23(64)$ & $35(73)$ & \\
\hline \multicolumn{4}{|c|}{ Family history of malignancy } \\
\hline Present & $15(42)$ & $35(73)$ & \multirow[t]{2}{*}{0.003} \\
\hline Absent & $21(58)$ & $13(27)$ & \\
\hline \multicolumn{4}{|l|}{ Stage $^{\mathrm{a}}$} \\
\hline I & $3(12)$ & $1(7)$ & \multirow[t]{4}{*}{0.39} \\
\hline II & $2(8)$ & $0(0)$ & \\
\hline III & $16(64)$ & $10(72)$ & \\
\hline IV & $4(16)$ & $3(21)$ & \\
\hline
\end{tabular}

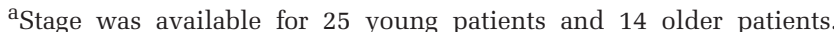
Patients with stage I-II and stage III-IV disease were combined for purposes of statistical analysis using the Fisher exact test.

with respect to different tumor types, however, demonstrated a higher prevalence of breast cancer among relatives of young patients with pleural mesothelioma than older patients $(40 \%$ vS $9 \%$; $P=0.02$; Supplementary Table S1). There was no difference in clinical stage between young patients and older patients with available clinical data.

\section{Histologic Characteristics}

A mean of eight hematoxylin and eosin slides (SD 7.4; range 1-23) were examined in young mesothelioma patients and three (SD 2.5; range 1-16) in older pleural mesothelioma patients. Histologic features of all mesotheliomas in each group are summarized in Table 2.

The majority $(78 \%)$ of pleural mesotheliomas in young patients were epithelioid, with a smaller number of biphasic $(19 \%)$ and sarcomatoid $(3 \%)$ tumors. While older patients more frequently had biphasic $(40 \%)$ or sarcomatoid (4\%) histology, the distribution of histologic types did not significantly differ between the study and comparison groups $(P=0.12)$. Given that non-epithelioid histology affects survival, we analyzed histologic charac- teristics between the study and control groups both among all histologic types as well as among epithelioid pleural mesotheliomas only. No histologic parameters were significantly different between the two groups among all histologic types $(P>0.05$ for all; Table 2). When considering epithelioid mesotheliomas only, younger patients were slightly more likely to demonstrate a tubulopapillary pattern and to have lymph node metastases; however, these differences were not statistically significant $(P>0.05$ for each; Table 2).

\section{Cytogenetics and Fluorescence In Situ Hybridization}

Karyotypic analysis was available in eight young patients and demonstrated deletions of chromosome 9 in one patient, chromosome 22 in four patients, and chromosome 3 in one patient, and translocations in two patients. Thirteen older patients had available karyotypes, demonstrating deletions of chromosomes 9 in seven patients, chromosome 22 in seven patients, chromosome 3 in two patients, and translocations in six patients. Genomic losses predominated over genomic gains in $50 \%$ of tumors in the study group compared with $62 \%$ of tumors in the comparison group. No recurrent translocations were seen among any patients in the study and comparison groups, and none of the translocations involved CDKN2A, NF2, or BAP1.

Fluorescence in situ hybridization results for both CDKN2A and NF2 were available for 18 young patients. Fluorescence in situ hybridization for $C D K N 2 A$ was available in 35 older patients, and for NF2 in 33 older patients (Table 3). CDKN2A deletion was significantly less frequent in younger than in older patients $(17 \%$ vs $54 \% ; P=0.01)$. No difference in the prevalence of NF2 deletion was seen between the two groups $(67 \%$ vs $78 \%$; $P=0.53$ ). Comparison of CDKN2A and NF2 status among epithelioid mesotheliomas between the study and control groups demonstrated similar results (Table 3).

\section{BAP1 Immunohistochemistry}

Young patients demonstrated less frequent loss of nuclear BAP1 immunohistochemical staining compared with older patients, but the difference was not statistically significant, regardless of histologic type $(P>0.05$; Table 3$)$. Next-generation sequencing in the group of older patients showed BAP1 alterations consisting of point mutations, insertions, deletions, copy number variations, or gene rearrangements in 19 of 21 cases with complete absence of BAP1 protein. With the exception of two cases that demonstrated missense mutations of unknown significance, any amount of BAP1 nuclear staining regardless of intensity or distribution was associated with wild-type $B A P 1$ gene status. Two cases with wild-type $B A P 1$ gene status demonstrated absence of 
Table 2 Histologic characteristics of mesothelioma in study patients

\begin{tabular}{|c|c|c|c|c|c|c|}
\hline \multirow{2}{*}{ Characteristic } & \multicolumn{2}{|c|}{ All histologic types } & \multirow[b]{2}{*}{ P-value } & \multicolumn{2}{|c|}{ Epithelioid mesotheliomas } & \multirow[b]{2}{*}{ P-value } \\
\hline & $\begin{array}{c}\text { Patients } \leq 35 \\
\mathrm{n}=36(\%)\end{array}$ & $\begin{array}{c}\text { Patients }>35 \\
\mathrm{n}=48(\%)\end{array}$ & & $\begin{array}{c}\text { Patients } \leq 35 \\
\mathrm{n}=28(\%)\end{array}$ & $\begin{array}{c}\text { Patients }>35 \\
\mathrm{n}=27(\%)\end{array}$ & \\
\hline \multicolumn{7}{|l|}{ Histologic type } \\
\hline Epithelioid & $28(78)$ & $27(56)$ & 0.12 & - & - & - \\
\hline Mixed & 7 (19) & $19(40)$ & & - & - & -- \\
\hline Sarcomatoid & $1(3)$ & $2(4)$ & & - & - & - \\
\hline \multicolumn{7}{|c|}{ Lymphovascular invasion } \\
\hline Present & $12(33)$ & $8(17)$ & 0.12 & $9(32)$ & $3(11)$ & 0.10 \\
\hline Absent & $24(67)$ & $40(83)$ & & $19(68)$ & $24(89)$ & \\
\hline \multicolumn{7}{|c|}{ Lymph node metastases } \\
\hline Present & $15(42)$ & $11(23)$ & 0.95 & $14(50)$ & $6(22)$ & 0.05 \\
\hline Absent & $21(58)$ & $37(77)$ & & $14(50)$ & $21(78)$ & \\
\hline \multicolumn{7}{|c|}{ Predominant growth pattern } \\
\hline Tubulopapillary & $15(42)$ & $11(23)$ & 0.26 & $15(54)$ & $7(26)$ & 0.15 \\
\hline Papillary & $1(3)$ & $1(2)$ & & $2(7)$ & $1(4)$ & \\
\hline Solid & $19(52)$ & $32(67)$ & & $10(36)$ & $17(63)$ & \\
\hline Micropapillary & $1(3)$ & $1(2)$ & & $1(3)$ & $1(4)$ & \\
\hline Other ${ }^{\mathrm{a}}$ & $0(0)$ & $3(6)$ & & $0(0)$ & $1(4)$ & \\
\hline \multicolumn{7}{|l|}{ Nuclear grade } \\
\hline Low & $8(22)$ & $7(15)$ & 0.09 & $7(25)$ & $3(11)$ & 0.34 \\
\hline Intermediate & $19(53)$ & 36 (75) & & $17(61)$ & $21(78)$ & \\
\hline High & $9(25)$ & $5(10)$ & & $4(14)$ & $3(11)$ & \\
\hline \multicolumn{7}{|l|}{ Mitotic Index } \\
\hline$>4$ & $13(36)$ & $14(29)$ & 0.64 & $6(21)$ & $7(26)$ & 0.76 \\
\hline$\leq 4$ & $23(64)$ & $34(71)$ & & $22(79)$ & $20(74)$ & \\
\hline
\end{tabular}

${ }^{\mathrm{a} O t h e r ~ p a t t e r n s ~ i n c l u d e d ~ t u m o r s ~ w i t h ~ a d e n o m a t o i d ~ a n d ~ p r o m i n e n t ~ m y x o i d ~ f e a t u r e s . ~}$

Table 3 Genetic features of mesothelioma in study patients

\begin{tabular}{|c|c|c|c|c|c|c|}
\hline \multirow{2}{*}{ Genetic Abnormality } & \multicolumn{2}{|c|}{ All histologic types } & \multirow[b]{2}{*}{ P-value } & \multicolumn{2}{|c|}{ Epithelioid mesotheliomas } & \multirow[b]{2}{*}{$\mathrm{P}$-value } \\
\hline & $\begin{array}{c}\text { Patients } \leq 35 \\
\mathrm{n}=36(\%)\end{array}$ & $\begin{array}{c}\text { Patients }>35 \\
\mathrm{n}=48(\%)\end{array}$ & & $\begin{array}{c}\text { Patients } \leq 35 \\
\mathrm{n}=28(\%)\end{array}$ & $\begin{array}{c}\text { Patients }>35 \\
\mathrm{n}=27(\%)\end{array}$ & \\
\hline \multicolumn{7}{|l|}{$C D K N 2 A(P 16)^{\mathrm{a}}$} \\
\hline Deletion & $3(17)$ & $19(54)$ & 0.01 & $2(14)$ & $12(60)$ & 0.01 \\
\hline No deletion & $15(83)$ & $16(46)$ & & $12(86)$ & $8(40)$ & \\
\hline \multicolumn{7}{|l|}{$N F 2^{\mathrm{a}}$} \\
\hline Deletion & $12(67)$ & $25(76)$ & 0.53 & $9(64)$ & $15(79)$ & 0.44 \\
\hline No deletion & $6(33)$ & $8(24)$ & & $5(36)$ & $4(21)$ & \\
\hline \multicolumn{7}{|l|}{ BAP1 expression } \\
\hline Lost & $8(22)$ & $21(44)$ & 0.06 & $8(29)$ & $12(44)$ & \\
\hline Retained & $28(78)$ & $27(56)$ & & $20(71)$ & $15(56)$ & 0.27 \\
\hline
\end{tabular}

${ }^{\mathrm{a} C o m p l e t e ~ F I S H ~ d a t a ~ w e r e ~ a v a i l a b l e ~ i n ~} 18$ of 36 young patients in the young cohort, including 14 epithelioid tumors; FISH data regarding CDKN2A and NF2 status were available in 35 and 33 older patients, respectively, including 20 and 19 patients with epithelioid mesothelioma.

BAP1 staining. Complete absence of BAP1 protein expression had a sensitivity of $90 \%$, specificity of $93 \%$, positive predictive value of $90 \%$, and negative predictive value of $93 \%$ for underlying $B A P 1$ genomic abnormalities, supporting the use of BAP1 immunohistochemistry as an indicator of $B A P 1$ alteration.

\section{Survival Analysis}

Median overall survival among all histologic types of pleural mesothelioma was 40 months in young patients and 26 months in older patients $(P=0.10$; Figure 2a). Median follow-up was 37 months in young patients and 31 months in older patients alive at the 

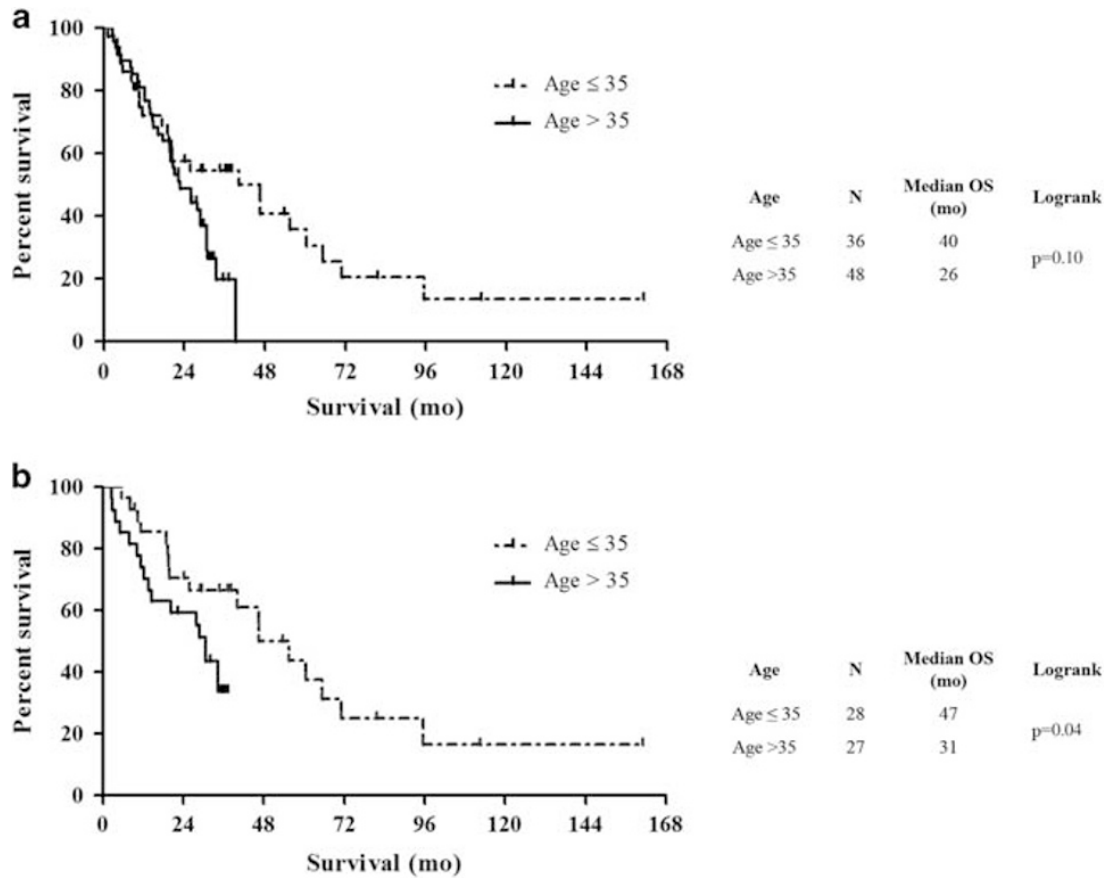

Figure 2 Overall survival in all pleural mesothelioma histologic types (a) and epithelioid pleural mesotheliomas only (b) among patients aged 35 or younger compared with a comparison group of patients aged greater than 35 .

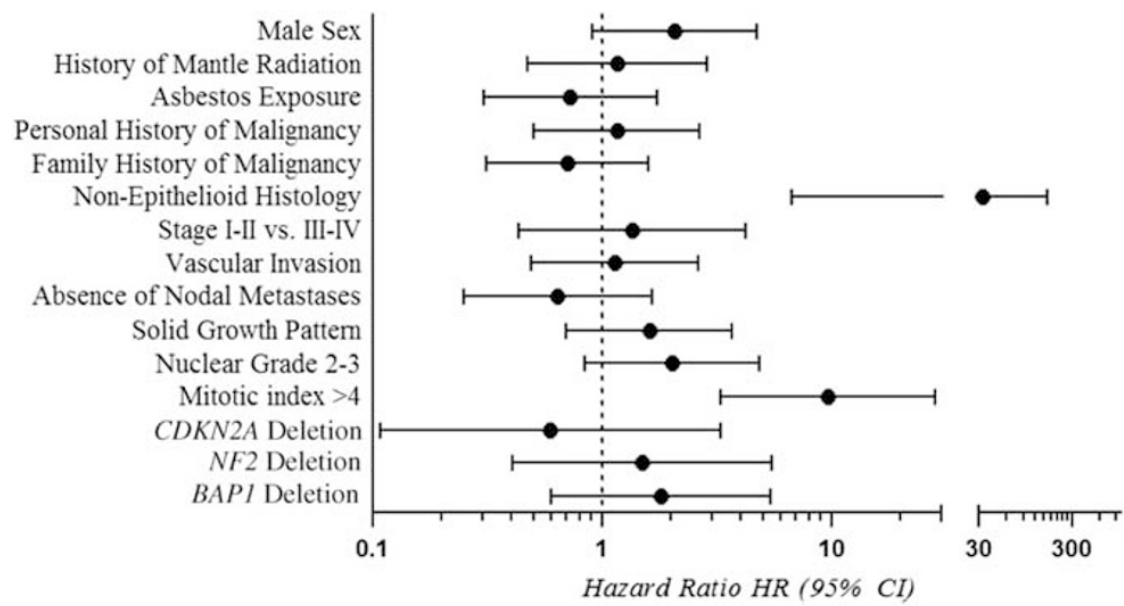

Figure 3 Overall survival in relation to clinicopathologic and genetic characteristics among young patients with pleural diffuse malignant mesothelioma.

end of the study period. Considering epithelioid mesotheliomas alone, overall survival was significantly longer in the study group than in the control group ( 47 vs 31 months; $P=0.04$; Figure 2b). Nonepithelioid histology and mitotic index $>4$ were significantly associated with a shorter overall survival among young patients (Figures 3 and 4 and Supplementary Table S2). No other histologic characteristics correlated with overall survival.

\section{Discussion}

Young patients with pleural mesothelioma are more frequently women and have longer survival compared with older patients, suggesting potential biological differences between tumors in these two groups. ${ }^{6,7,10}$ The higher incidence of pleural mesothelioma among men in older patients may be related to occupational asbestos exposure, given that men and women who have been exposed to equivalent amounts of asbestos have a similar incidence of pleural mesothelioma. ${ }^{21}$ Overall survival in pleural mesothelioma has been reported to be better in patients who have not been exposed to asbestos, and young women are disproportionately represented among long-term survivors. ${ }^{22,23}$ Furthermore, equal sex distribution, young age, and improved survival have been reported among patients with pleural mesothelioma in populations 

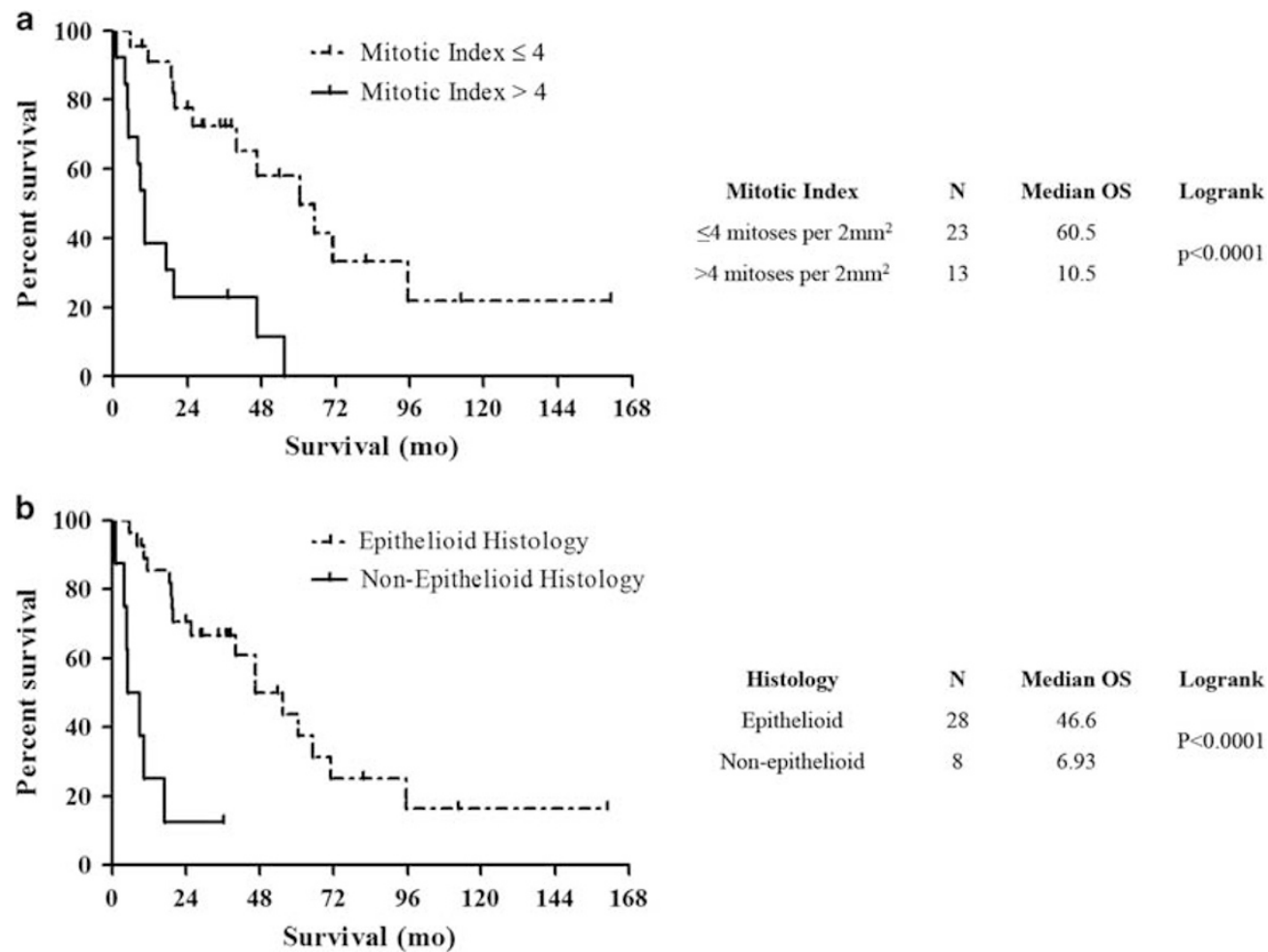

$\begin{array}{cccc}\text { Histology } & \text { N } & \text { Median OS } & \text { Logrank } \\ \text { Epithelioid } & 28 & 46.6 & \\ \text { Non-epithelioid } & 8 & 6.93 & \mathrm{P}<0.0001\end{array}$

Figure 4 Overall survival in patients aged 35 or younger with pleural diffuse malignant mesothelioma is significantly associated with mitotic index (a) and histologic type (b).

exposed to naturally occurring non-asbestos fibers, therapeutic radiation, and other potentially carcinogenic materials. ${ }^{24-26}$ These observations have raised the possibility that pleural mesothelioma in young patients may be related to different etiologic factors than in older patients, and may therefore possess different clinical, histologic, and genetic characteristics that could have potential impact on patient prognosis and treatment.

The current study is the largest comprehensive clinical, histologic, genetic, and survival analysis of young pleural mesothelioma patients to date. Our findings confirm an equivalent sex distribution and significantly lower rates of reported asbestos exposure in pleural mesothelioma patients aged 35 and younger compared with older patients. A history of therapeutic mantle radiation for Hodgkin lymphoma was present in a quarter of young pleural mesothelioma patients (Table 1). This finding confirms the reported etiologic association between therapeutic radiation and pleural mesothelioma, ${ }^{26,27}$ and suggests that therapeutic radiation may be a particular risk factor for pleural mesothelioma in this population. Young and older pleural mesothelioma patients did not differ with respect to personal history of malignancy. One would expect younger patients to have a significantly lower prevalence of prior malignancy than older patients; while this finding can partially be explained by the comparatively high prevalence of Hodgkin lymphoma in the young pleural mesothelioma group, 5 of 36 young patients had a personal history of other malignancies, suggesting that other factors (eg genetic predisposition) may be contributory. Family history of malignancy was significantly more prevalent among older than among young pleural mesothelioma patients. This result, however, is likely explained by age-related increases in cancer among family members of older mesothelioma patients, and not due to inherited cancer susceptibility. Interestingly, there was a much higher relative prevalence of family history of breast cancer among young than among older pleural mesothelioma patients (Supplementary Table S1), and one patient in the study group had a personal history of invasive ductal carcinoma of the breast at age 28 in the setting of a germline BRCA2 mutation. These findings, in the absence of a higher overall incidence of family cancer history compared with older patients, also suggests that young patients with pleural mesothelioma may have an underlying susceptibility to other specific types of malignancy.

There were no statistical differences in the distribution of histologic types of pleural mesothelioma, presence of lymphovascular invasion, nodal metastases, growth patterns, nuclear grade, or mitotic index among all mesotheliomas between young and older patients. Although the difference in distribution of histologic mesothelioma types did not reach significance between study and comparison groups, the higher prevalence of biphasic and sarcomatoid mesotheliomas in the comparison group prompted 
us to examine the remaining histologic and genetic characteristics in epithelioid mesotheliomas separately, as non-epithelioid histology is associated with poor survival and a higher prevalence of CDKN2A alterations. Young patients with epithelioid mesotheliomas demonstrated a slightly higher prevalence of lymph node metastases and tubulopapillary growth that did not reach statistical significance, and were otherwise similar to older patients.

Approximately $43-80 \%$ of all pleural diffuse malignant mesotheliomas are reported to harbor deletions of the CDKN2A gene and $40-65 \%$ have NF2 deletions. ${ }^{28-30}$ More recently, somatic alterations of the BAP1 gene have also been described in $42-80 \%$ pleural mesothelioma patients, and are independent of CDKN2A and NF2 alterations. ${ }^{31,32}$ $C D K N 2 A$ deletion is reported to be associated with asbestos exposure in vitro and in vivo, and has been associated with poor prognosis in multiple studies compared with pleural mesotheliomas without the deletion. ${ }^{30,33,34}$ Somatic BAP1 abnormalities have not consistently been shown to be associated with asbestos exposure, and have shown variable correlation with overall survival. ${ }^{25,35}$ The prognostic significance of NF2 alterations has not been studied in depth. $54 \%$ of pleural mesotheliomas in older patients in our study had CDKN2A deletions, and $76 \%$ had losses at the NF2 locus. While the prevalence of NF2 deletion in the study group was not significantly different from the older comparison group, young patients had a much lower prevalence of $C D K N 2 A$ deletion. Given that germline mutations of the BAP1 gene confer susceptibility to a number of tumors, including pleural diffuse malignant mesothelioma, ${ }^{36}$ we also evaluated the possibility that $B A P 1$ alterations may play an important oncogenic role in young pleural mesothelioma patients. As genomic sequencing data were not available in the study group, we compared BAP1 immunohistochemistry to $B A P 1$ gene sequencing data in the comparison group and demonstrated a high sensitivity and specificity of BAP1 immunohistochemistry for detection of somatic $B A P 1$ genetic abnormalities. Interestingly, tumors in the study group demonstrated loss of BAP1 expression less frequently than tumors from older patients $(22 \%$ vs $44 \%$ ), but the difference did not reach statistical significance $(P=0.06)$.

Although the lower prevalence of $C D K N 2 A$ deletion among young patients could potentially be attributed to a lower prevalence of non-epithelioid histology in the study group, this genomic difference persisted between the study and comparison groups even when epithelioid mesotheliomas were analyzed separately. Additionally, while the number of cases with available karyotypic analysis in the current study were few, mesotheliomas in young patients had a slightly lower prevalence of genomic losses than did older patients. In combination with our fluorescence in situ hybridization and immunohistochemical results, this suggests a genomic landscape among young pleural mesothelioma patients that has been recently described to be associated with therapeutic radiation rather than asbestos exposure. ${ }^{37}$ These findings support the concept that differences in the biology of pleural mesothelioma between young and older patients could be related to radiation as a major causative agent in the former, compared with asbestos exposure in the latter.

Young patients had a median overall survival of 40 months, approximately 16 months longer than the median overall survival in the comparison group. Although the difference was not statistically significant, due to small sample size, this finding aligns with prior reports that young patients survive longer than older patients. ${ }^{7,38,39}$ Five-year survival among young patients in the current study was $31 \%$, higher than the reported rates of $12-15 \%$ among all pleural mesothelioma patients, ${ }^{40,41}$ and including two patients who were alive at the last time of followup at 112 and 161 months. Notably, in our separate analysis of epithelioid tumors only, overall survival was significantly longer among young patients compared with older patients. This suggests that there may be a subset of exceptionally long survivors among young patients with epithelioid pleural diffuse malignant mesothelioma. Tumor histology and mitotic index were the only pathologic variables that correlated with overall survival in our study group, and confirm prior reports that mitotic index $>4$ and non-epithelioid histology confer poor prognosis in pleural diffuse malignant mesothelioma. ${ }^{15,38}$ In contrast to some studies, ${ }^{15}$ low nuclear grade did not significantly correlate with a better overall survival in the study group.

The primary limitations of our study are the small number of patients in the study and control groups due to the low prevalence of mesothelioma in young patients and selection criteria for the comparison group, respectively, the absence of asbestos fiber counts in our study and comparison groups as an objective measure of exposure, and the need to use immunohistochemistry as a surrogate for $B A P 1$ gene alterations in the study group. Additionally, since our control group was selected on the basis of availability of next-generation sequencing results, the time period during which older patients were treated was more limited and recent than the study group time period, raising the possibility that there were differences in patient management and treatment efficacy between the groups that could affect survival analysis.

Our findings must be interpreted within the context of these limitations; nevertheless, the distribution of demographic data and histologic types of mesothelioma in our study and comparison groups are in agreement with prior epidemiologic characterizations of these patient populations, ${ }^{5-7}$ and our results therefore still suggest a number of interesting conclusions that merit further study. Young patients had an equal sex distribution, were 
less likely to report asbestos exposure, and had a different genomic profile than our older comparison group, confirming similar prior reports ${ }^{7,9,10,13-14,37,42}$ and suggesting that other pathogenetic pathways may play a greater role in this demographic group than in older patients. Specifically, mantle radiation, which has previously been associated with malignant mesothelioma and a distinct genomic profile similar to tumors in our study group,,$^{26,27}$ was significantly more prevalent in our study group and may represent one such etiologic agent. Among young patients with pleural mesothelioma, a higher prevalence of family history of breast cancer, lower prevalence of $C D K N 2 A$ deletion, potentially lower prevalence of somatic $B A P 1$ alteration, and longer overall survival among epithelioid mesotheliomas suggest that pleural diffuse malignant mesothelioma in patients aged 35 years and younger has unique biological features and may be driven by different oncogenetic events than pleural diffuse malignant mesothelioma in older patients. Our results must be confirmed by studies of larger patient cohorts with systematic evaluation of genomic and clinical characteristics, but indicate that pleural diffuse malignant mesothelioma in young patients is associated with distinct clinical and genetic characteristics that may provide insight into the complex pathogenetic mechanisms of mesothelioma.

\section{Acknowledgments}

We acknowledge the Center for Advanced Molecular Diagnostics and William G Richards, $\mathrm{PhD}$ for their technical and intellectual contributions to this work.

\section{Disclosure/conflict of interest}

LRC serves on the Advisory board for Merck Sharp \& Dohme and undertakes medicolegal work related to mesothelioma and lung cancer. RB receives support from the National Cancer Institute, Verastem, Genentech-Roche, and Castle Biosciences via research grants to Brigham and Women's Hospital. These financial disclosures do not apply to the current study, which is not associated with a specific source of funding. The remaining author declare no conflict of interest.

\section{References}

1 Spirtas R, Heineman EF, Bernstein L, et al. Malignant mesothelioma: attributable risk of asbestos exposure. Occup Environ Med 1994;51:804-811.

2 Coggon D, Inskip $\mathrm{H}$, Winter $\mathrm{P}$, et al. Differences in occupational mortality from pleural cancer, peritoneal cancer, and asbestosis. Occup Environ Med 1995;52: 775-777.

3 Galateau-Salle F, Churg A, Roggli V et al. Diffuse malignant mesothelioma: epithelioid mesothelioma. In:
Travis WD, Brambilla E, Burke AP et al. (eds). WHO Classification of Tumours of the Lung, Pleura, Thymus and Heart. IARC: Lyon, France, 2015, pp 156-154.

4 Neumann V, Günthe S, Mülle KM, et al. Malignant mesothelioma-German mesothelioma register 1987-1999. Int Arch Occup Environ Health 2001;74:383-395.

5 Centers for Disease Control. Work-Related Lung Disease Surveillance System: Mesothelioma. Available at: http://wwwn.cdc.gov/eworld/Grouping/Malignant mesothelioma/100 (accessed 20 February 2017).

6 National Cancer Institute. Surveillance, Epidemiology, and End Results Program: Incidence-age distribution of incidence cases by site, 1975-2012. Available at: http://seer.cancer.gov/csr/1975_2012/results_single/sect_ 01_table.11_2pgs.pdf (accessed 25 February 2017, accessed 20 February 2017).

7 Thomas A, Chen Y, Yu T, et al. Distinctive clinical characteristics of malignant mesothelioma in young patients. Oncotarget 2015;6:16766-16773.

8 Delgermaa V, Takahashi K, Park E-K, et al. Global mesothelioma deaths reported to the World Health Organization between 1994 and 2008. Bull World Health Organ 2011;89:716-724.

9 Kane MJ, Chahinian aP, Holland JF. Malignant mesothelioma in young adults. Cancer 1990;65:1449-1455.

10 Fraire AE, Cooper S, Greenberg SD, et al. Mesothelioma of childhood. Cancer 1988;62:838-847.

11 Teta MJ, Mink PJ, Lau E, et al. US mesothelioma patterns 1973-2002: indicators of change and insights into background rates. Eur J Cancer Prev 2008;17: 525-534.

12 Moran CA, Albores-Saavedra J, Suster S. Primary peritoneal mesotheliomas in children: a clinicopathological and immunohistochemical study of eight cases. Histopathology 2008;52:824-830.

13 Grundy GW, Miller R. Malignant mesothelioma in childhood: report of 13 cases. Cancer 1972;30:1216-1218.

14 Brenner J, Sordillo PP, Magill GB. Malignant mesothelioma in children: report of seven cases and review of the literature. Med Pediatr Oncol 1981;9:367-373.

15 Kadota K, Suzuki K, Colovos C, et al. A nuclear grading system is a strong predictor of survival in epitheloid diffuse malignant pleural mesothelioma. Mod Pathol 2012;25:260-271.

16 Factor RE, Cin PD, Fletcher JA, et al. Cytogenetics and fluorescence in situ hybridization as adjuncts to cytology in the diagnosis of malignant mesothelioma. Cancer Cytopathol 2009;117:247-253.

17 Sholl LM, Do K, Shivdasani P, et al. Institutional implementation of clinical tumor profiling on an unselected cancer population. JCI Insight 2016;1:e87062.

18 Cibulskis K, Lawrence MS, Carter SL, et al. Sensitive detection of somatic point mutations in impure and heterogeneous cancer samples. Nat Biotechnol 2013; 31:213-219.

19 Pugh TJ, Amr SS, Bowser MJ, et al. VisCap: inference and visualization of germ-line copy-number variants from targeted clinical sequencing data. Genet Med 2016;18:712-719.

20 Abo RP, Ducar M, Garcia EP, et al. BreaKmer: detection of structural variation in targeted massively parallel sequencing data using kmers. Nucleic Acids Res 2015;43:e19-e19.

21 Reid A, Berry G, de Klerk N, et al. Age and sex differences in malignant mesothelioma after residential exposure to blue asbestos (Crocidolite). Chest 2007;131: 376-382. 
22 Law MR, Ward FG, Hodson ME, et al. Evidence for longer survival of patients with pleural mesothelioma without asbestos exposure. Thorax 1983;38:744-746.

23 Sugarbaker DJ, Wolf AS, Chirieac LR, et al. Clinical and pathological features of three-year survivors of malignant pleural mesothelioma following extrapleural pneumonectomy. Eur J Cardiothorac Surg 2011;40: 298-303.

24 Roushdy-Hammady I, Siegel J, Emri S, et al. Geneticsusceptibility factor and malignant mesothelioma in the Cappadocian region of Turkey. Lancet 2001;357: 444-445.

25 Baumann F, Flores E, Napolitano a, et al. Mesothelioma patients with germline BAP1 mutations have 7-fold improved long-term survival. Carcinogenesis 2014;36: 76-81.

26 Chirieac LR, Barletta JA, Yeap BY, et al. Clinicopathologic characteristics of malignant mesotheliomas arising in patients with a history of radiation for hodgkin and non-hodgkin lymphoma. J Clin Oncol 2013;31: 4544-4549.

27 Teta MJ, Lau E, Sceurman BK, et al. Therapeutic radiation for lymphoma risk of malignant mesothelioma. Cancer 2007;109:1432-1438.

28 Illei PB, Rusch VW, Zakowski MF, et al. Homozygous deletion of CDKN2A and codeletion of the methylthioadenosine phosphorylase gene in the majority of pleural mesotheliomas. Clin Cancer Res 2003;9:2108-2113.

29 Chiosea S, Krasinskas A, Cagle PT, et al. Diagnostic importance of 9p21 homozygous deletion in malignant mesotheliomas. Mod Pathol 2008;21:742-747.

30 Murthy SS, Testa JR. Asbestos, chromosomal deletions, and tumor suppressor gene alterations in human malignant mesothelioma. J Cell Physiol 1999;180: 150-157.

31 Bott M, Brevet M, Taylor BS, et al. The nuclear deubiquitinase BAP1 is commonly inactivated by somatic mutations and 3p21.1 losses in malignant pleural mesothelioma. Nat Genet 2011;43:668-672.

32 Yoshikawa Y, Sato A, Tsujimura T, et al. Frequent inactivation of the BAP1 gene in epithelioid-type malignant mesothelioma. Cancer Sci 2012;103:868-874.
33 Dacic S, Kothmaier $\mathrm{H}$, Land S, et al. Prognostic significance of p16/[CDKN2A] loss in pleural malignant mesotheliomas. Virchows Arch 2008;453:627-635.

34 López-Ríos F, Chuai S, Flores R, et al. Global gene expression profiling of pleural mesotheliomas: overexpression of Aurora kinases and P16/CDKN2A deletion as prognostic factors and critical evaluation of microarray-based prognostic prediction. Cancer Res 2006;66:2970-2979.

35 Zauderer MG, Bott M, McMillan R, et al. Clinical characteristics of patients with malignant pleural mesothelioma harboring somatic BAP1 mutations. J Thorac Oncol 2013;8:1430-1433.

36 Testa JR, Cheung M, Pei J, et al. Germline BAP1 mutations predispose to malignant mesothelioma. Nat Genet 2011;43:1022-1025.

37 Borczuk AC, Pei J, Tuab RN, et al. Genome-wide analysis of abdominal and pleural malignant mesothelioma with DNA arrays reveals both common and distinct regions of copy number alteration. Cancer Biol Ther 2016;17:328-335.

38 Antman K, Shemin R, Ryan L, et al. Malignant mesothelioma: prognostic variables in a registry of 180 patients, the Dana-Farber Cancer Institute and Brigham and Women's Hospital experience over two decades, 1965-1985. J Clin Oncol 1988;6:147-153.

39 Spirtas R, Connelly RR, Tucker MA. Survival patterns for malignant mesothelioma. Int J Cancer 1988;41: $525-530$

40 Sugarbaker DJ, Flores RM, Jaklitsch MT, et al. Resection margins, extrapleural nodal status, and cell type determine postoperative long-term survival in trimodality therapy of malignant pleural mesothelioma: results in 183 patients. J Thorac Cardiovasc Surg 1999;117:54-63.

41 Stathopoulos J, Antoniou D, Stathopoulos GP, et al. Mesothelioma: treatment and survival of a patient population and review of the literature. Anticancer Res 2005;25:3671-3676.

42 McDonald JC, Edwards CW, Gibbs AR, et al. Case-referent survey of young adults with mesothelioma: II. Occupational analyses. Ann Occup Hyg 2001;45:519-523.

Supplementary Information accompanies the paper on Modern Pathology website (http://www.nature.com/ modpathol) 